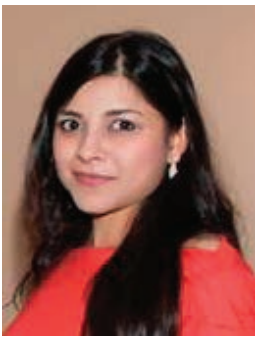

\title{
PREDICTORS AND MANAGEMENT OF RIGHT HEART FAILURE AFTER LEFT VENTRICULAR ASSIST DEVICE IMPLANTATION
}

\author{
Nadia Fida, M.D.; Matthias Loebe, M.D.; Jerry D. Estep, M.D.; Ashrith Guha, M.D., M.P.H. \\ Houston Methodist DeBakey Heart \& Vascular Center, Houston Methodist Hospital, Houston, Texas
}

N. Fida, M.D.

\begin{abstract}
Newer generation continuous-flow left ventricular assist devices (LVADs) have overall improved outcomes with lower incidences of right ventricular failure (RVF) than their pulsatile predecessors, yet RVF still occurs in 9\% to 40\% of LVAD recipients. Postimplant, RVF is associated with poor outcomes, end-organ dysfunction, high mortality, and reduced survival to transplant. Therefore, preoperative risk stratification, appropriate patient selection, and optimal timing of implant are of paramount importance. In this article, we review the definition, incidence, pathophysiology, and current risk prediction models for RVF and touch on the contemporary management of RVF perioperatively and post-LVAD implant.
\end{abstract}

\section{Scope of the Problem}

Early recognition and improved outcomes of ischemic heart disease has resulted in an aging population and a global increase in the prevalence of heart failure (HF). ${ }^{1}$ Consequently, the burden of stage D heart failure (HF), which is unresponsive to conventional medical therapy, has surpassed the availability of the finite donor organ pool. ${ }^{2}$ Significant advances in the field of mechanical circulatory support has made destination therapy (DT) with left ventricular assist devices (LVADs) a promising alternative to heart transplantation, with 1-year postimplant actuarial survival at about $80 \%$ with a continuous flow LVAD (CF-LVAD). ${ }^{3}$ However, despite newer technology and greater familiarity with patient management, physicians continue to be confronted by early and/or late right ventricular failure (RVF) that significantly impacts survival post-LVAD implantation. As none of the current mechanical circulatory support (MCS) devices are approved for DT for solitary right heart support or biventricular support, it is critical to identify preoperative predictors of right heart failure post-LVAD implant.

\section{Definition and Incidence of Right Ventricular Failure after LVAD Placement}

In the absence of a universally agreed-upon definition of RVF after LVAD placement, its reported incidence varies with the institutional practice, the United Network for Organ Sharing (UNOS) indication for MCS, the device type, and the criteria used to define failure. Additionally, it is now recognized that RVF is a progressive condition that can occur beyond the immediate postoperative period. However, this emerging concept of "late RVF" remains ill-defined and difficult to predict. The Interagency Registry for Mechanically Assisted Circulatory Support (INTERMACS) defines RVF as persistent signs and symptoms of RV dysfunction evident by central venous pressure $(\mathrm{CVP})>18$ $\mathrm{mm} \mathrm{Hg}$ with a cardiac index $(\mathrm{CI})<2.0 \mathrm{~L} / \mathrm{min} / \mathrm{m}^{2}$ in the absence of increased left atrial filling pressure/pulmonary capillary wedge pressure $(\mathrm{PCWP})>18 \mathrm{~mm} \mathrm{Hg}$, cardiac tamponade, ventricular arrhythmias, and/or pneumothorax requiring either right ventricular assist device (RVAD) implantation or inhaled nitric oxide or inotropic therapy for $\geq 14$ days after LVAD implantation. ${ }^{4}$ According to INTERMACS, RVF severity is described as follows:

- Severe, when there is a need for RVAD;

- Moderate, when inotropes or intravenous or inhaled pulmonary vasodilators are used; and

- Mild, when a combination of $\geq 2$ signs and symptoms are present but without the need for RVAD or inotropic and/ or vasodilator support. Signs and symptoms include CVP > $18 \mathrm{~mm} \mathrm{Hg}, \mathrm{CI}<2.3 \mathrm{~L} / \mathrm{min} / \mathrm{m}^{2}$, ascites, moderate to severe peripheral edema, or evidence of high CVP on physical exam or transthoracic echocardiogram.

The incidence of severe RVF necessitating RVAD placement after LVAD or preemptive BiVAD support is reported to be anywhere from $9.4 \%$ to $37.0 \% .^{5-9}$ Most studies have reported the incidence encompassing the need for inotropic support with or without RVAD ( $\geq 14$ days) after LVAD implant, with a range of between $20.2 \%$ to $44 \%{ }^{10-12}$ We know that prolonged RV failure (i.e., extended inotrope support) for more than 2 weeks is associated with adverse subsequent outcomes. Given the lack of a standardized definition for late RVF, the long-term implications are unknown. However, we believe it is associated with increased morbidity based on symptoms and HF-related readmissions. This has a bearing not only on long-term survival of the DT population but also on quality of life and utility of resources and cost.

\section{Pathophysiology of Right Ventricular Failure}

Biventricular dysfunction is a common end point for stage $\mathrm{D}$ heart failure where chronically elevated left-sided filling pressures have induced high pulmonary vascular resistance (PVR), resulting in secondary pulmonary hypertension and subsequently RVF. Acute unloading of the left ventricle seen after successful LVAD implantation drops the PCWP, thereby relieving congestion, and 
Acute RV Failure
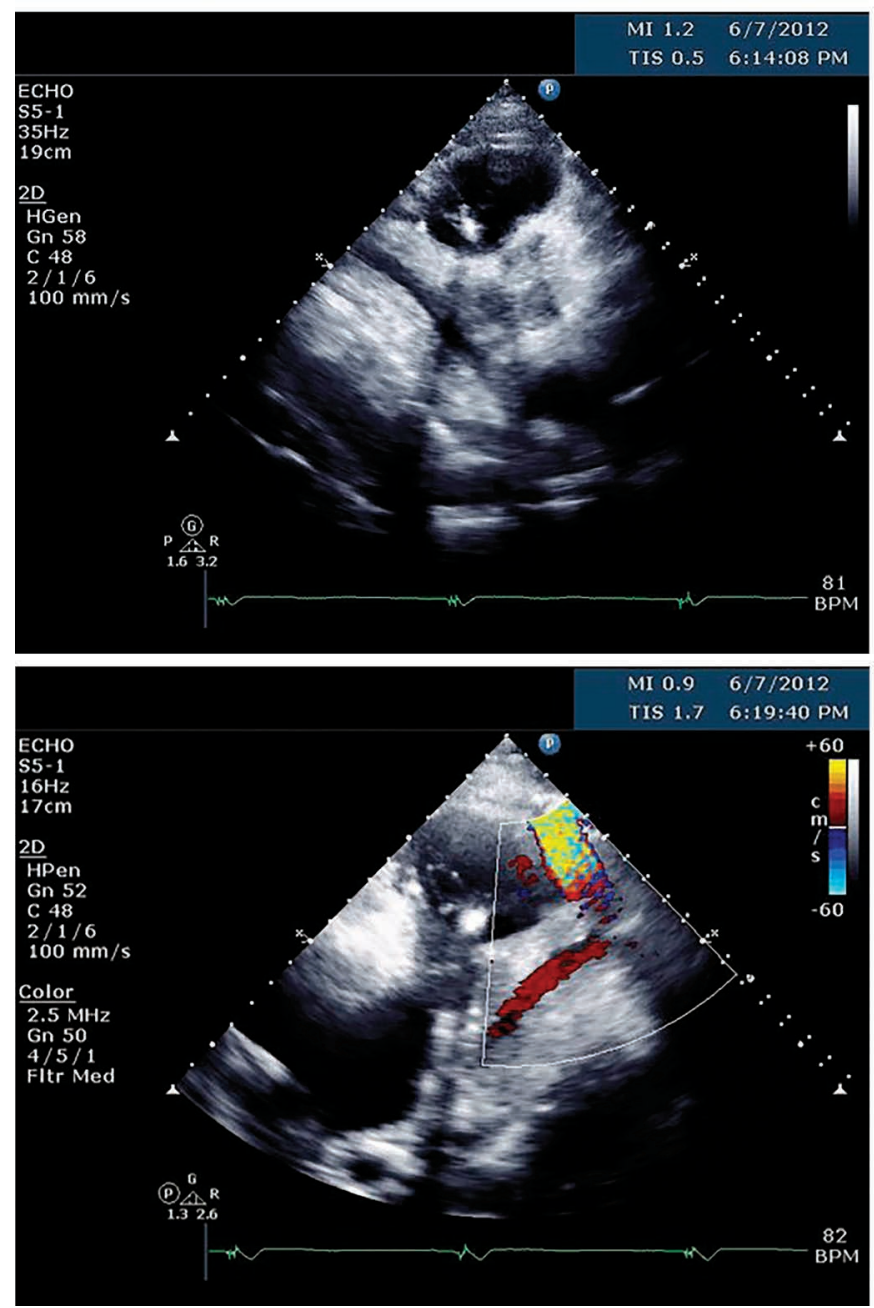

Resolved Overt RV Failure
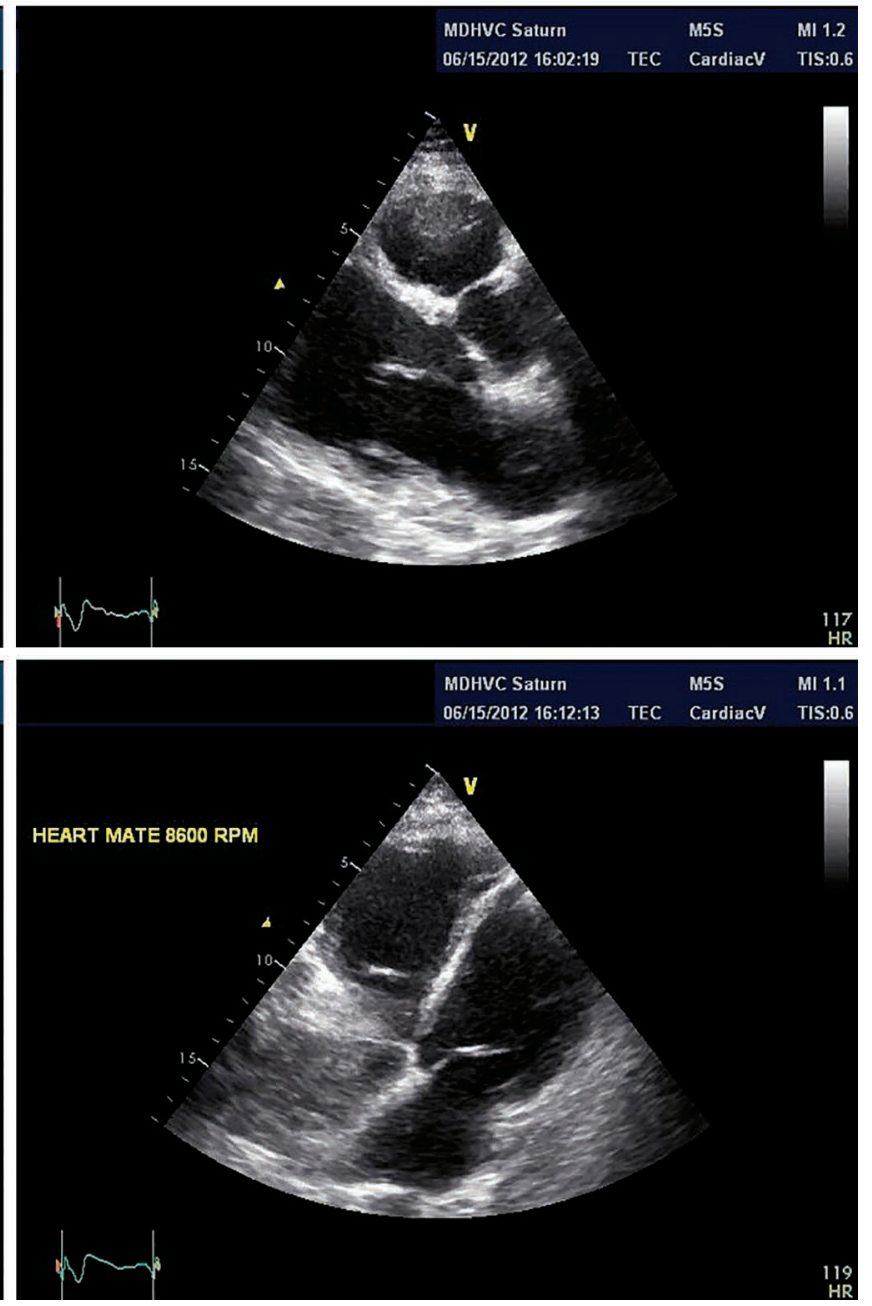

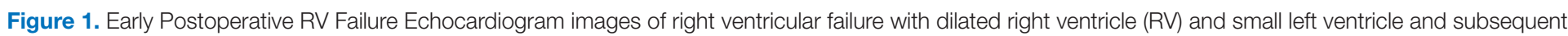
recovery of RV function.

recovers cardiac output. Kukucka et al. recently confirmed these findings in a perioperative transesophageal echocardiography

(TEE) study with improved RV end-diastolic geometry resulting from marked reduction in RV afterload. ${ }^{13}$

The pathophysiology of RV failure is linked to increased preload, decreased contractility, and increased afterload, each of which can occur alone or in combination in the perioperative period. Improved left-sided forward flow with mechanical unloading not uncommonly exacerbates the RV preload in conjunction with perioperative transfusions of blood products. This not only alters the Frank-Starling mechanics for the deconditioned RV but also leads to worsening of tricuspid regurgitation (TR), leftward bowing of the septum, and ventriculo-arterial uncoupling (Figure 1). Several hemodynamic and mechanical factors-for example, proper positioning of the inflow cannula and optimal speed to avoid suction events-affect septal interdependence after LVAD implant and can affect RV function. In a small study, Topilsky et al. ${ }^{14}$ showed that persistent inefficient unloading conditions and leftward shift of the interatrial septum caused persistence of increased RV afterload and was associated with worse 90-day outcomes post-LVAD implant. A cardiovascular-respiratory modeling study using an implantable rotary blood pump demonstrated that reduced septal contribution with increasing pump speeds resulted in elevated RV work and mechanical dyssynchrony. ${ }^{15}$

\section{Risk Factors and Risk Prediction Models for RVF Postimplantation}

Preoperative RV function is an established prognosticator of RVF post-LVAD implant. Existing risk prediction models are derived from retrospective studies using demographics, presence of end-organ dysfunction, hemodynamics, and prior open chest procedures. Few studies have incorporated contemporary imaging techniques for risk prediction. Notably, most scores are derived from patient populations supported with earlier-generation pulsatile-flow pumps and hence are not fully representative of the present-day LVAD population. The usefulness of RVF risk prediction models is limited primarily because of their modest discrimination in the derivation cohort (Table 1). There has been only one small independent validation study to date on the CF-LVAD population that compared all scores and demonstrated that no single score could predict the need for RVAD.

As incorporated in the INTERMACS definition, most of the hemodynamic factors are strong risk factors for postimplant RVF, but no one factor has consistently shown to 

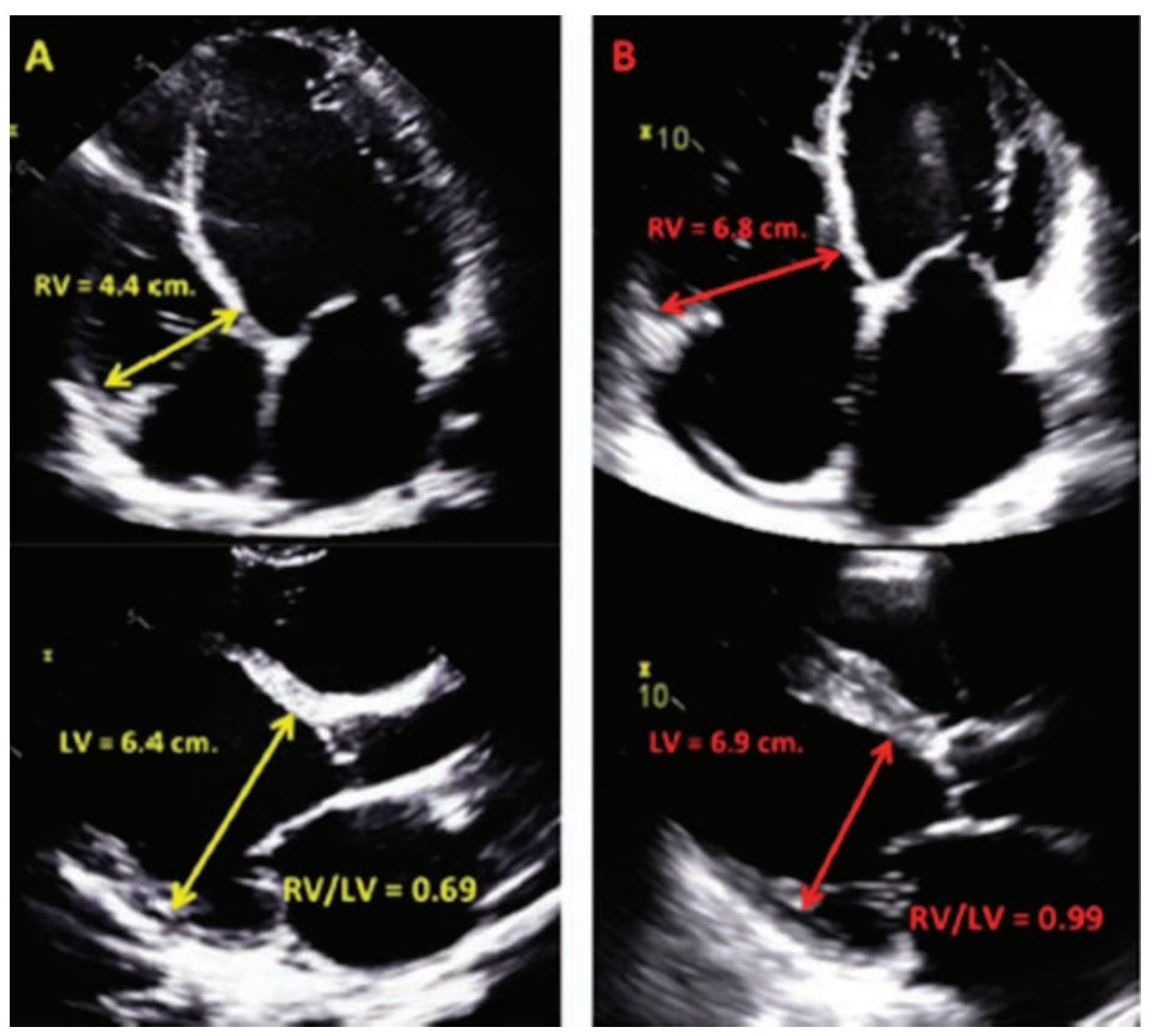

Figure 2. The ratio of right ventricle (RV) to left ventricle in prediction of postoperative RV failure.

be a significant predictor in multivariate analyses. Reverse remodeling of pulmonary vasculature can potentially occur by continuing unloading, and, unlike for heart transplantation, elevated pulmonary vascular resistance does not predict postimplant RVF.711,12 Surrogates of reduced RV contractility, i.e., low pulmonary artery systolic pressure, and RV stroke work index remain markers of risk but have not yielded substantial predictive information. $.^{8,10,12,16} \mathrm{~A} C \mathrm{CV} / \mathrm{PCWP}$ ratio $>0.63$ was shown to be an independent predictor of early RVF risk in the HeartMate II Bridge-to-Transplantation Pivotal Trial population with overall low discrimination (0.68).

Echocardiography is emerging as a feasible tool to evaluate parameters of RV dysfunction. ${ }^{17}$ Puwanant et al. ${ }^{18}$ demonstrated that tricuspid annular plane systolic excursion (TAPSE) with a cut-off value of $7.5 \mathrm{~mm}$ was $91 \%$ specific and $46 \%$ sensitive in predicting post-LVAD RVF. Low TAPSE as a marker of RV longitudinal function has been considered in many studies but not established as a predictor. . $^{13,16,19,20}$ The reason is that TAPSE is only a regional marker of RV function, i.e., it assumes that the motion of the RV free wall base represents the function of other segments. Reduced (<35\%) RV fractional area change (RVFAC) predicted RVF in a small retrospective study, ${ }^{19}$ but this has not been reproduced in another study. The $\mathrm{RV} / \mathrm{LV}$ diameter ratio as a surrogate of disproportionate RV remodeling analogous to CVP/PCWP ratio showed a strong association with RVF in some studies. ${ }^{13,21}$ Vivo et al. ${ }^{21}$ demonstrated that RVFAC did not predict RVF, but, based on receiver operating characteristic curve analysis, an $\mathrm{RV} / \mathrm{LV}$ diameter ratio $>0.75(\mathrm{AUC}=0.68)$ was as optimal as the Matthews $(\mathrm{AUC}=0.69)^{11}$ and Kormos $(\mathrm{AUC}=0.63)^{12}$ risk scoring systems in predicting RVF alone and the composite of RVF and death (Figure 2).

Aissoui et al. ${ }^{22}$ calculated RVFAC, mitral E velocity, basal RV end diastolic diameter, RV tissue Doppler velocity, and TAPSE and showed that the combined echo score predicts RVF with $92 \%$ sensitivity and $67 \%$ specificity.

Strain, strain rate, and speckle tracking by echocardiography are evolving as promising approaches for evaluating RV function. A recent report by Grant et al. ${ }^{16}$ indicated that decreased RV peak longitudinal strain was a novel predictor of RVF, whereas RV/LV ratio by TTE was not. Strain- or strain ratebased indices have not been reproduced as predictors of RVF by other authors. 


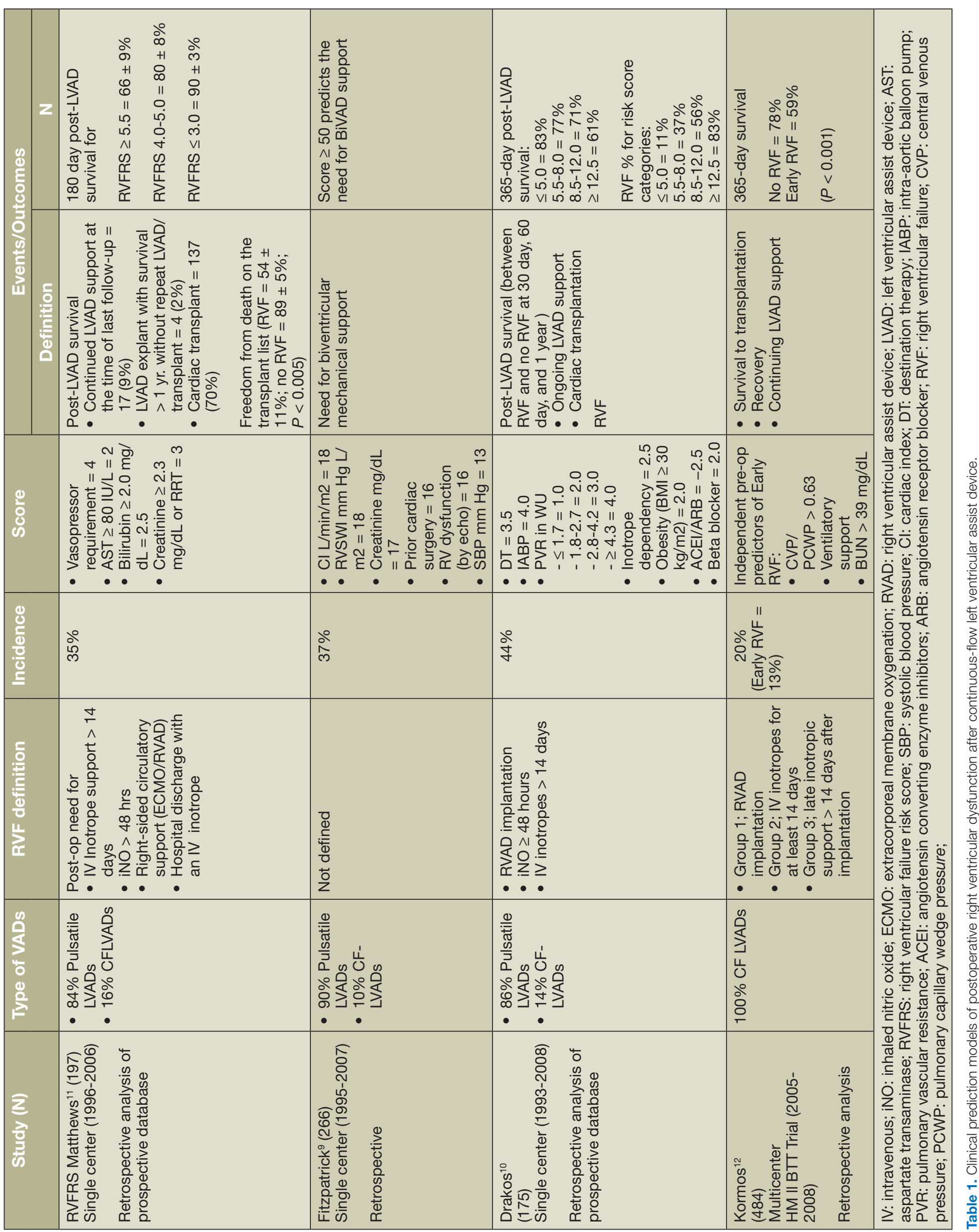


Given the lack of consensus thus far regarding the predictive value of any single imaging parameter, an aggregate assessment using relevant left-sided (e.g,. indexed LV size) and right-sided parameters (e.g., RV parameters described above, TR severity, ${ }^{23}$ and right atrial pressure) is likely the best approach.

\section{Management of RVF}

Appropriate patient selection, optimal timing of implant, and preoperative optimization of comorbid conditions are the key steps towards successful LVAD placement and minimizing the incidence of RVF. Early identification and management of RVF in the preoperative period and detection of its late manifestation are critical in decreasing mortality and morbidity in patients supported by CF-LVADs.

\section{Medical Management}

A pulmonary artery catheter-tailored management to optimize hemodynamics and volume status of the patient is an ideal approach in the postoperative period. A goal CVP $<15 \mathrm{~mm} \mathrm{Hg}$ ensures decreased RV workload and hepatic and renal congestion. Inotropes, vasodilators, and ultrafiltration should be considered for CVP > $15 \mathrm{~mm} \mathrm{Hg.}{ }^{24}$ Besides pharmacological therapy, measures to reduce PVR by adequate ventilation to reduce hypoxia, hypercarbia, and acidosis are imperative.

\section{Pulmonary Vasodilators}

Inhaled nitric oxide (iNO) is a selective pulmonary vasodilator that successfully reduces PVR. A prospective, randomized, doubleblind, placebo-controlled, multicenter trial demonstrated that iNO initiated before weaning from cardiopulmonary bypass (CPB) and continued for 48 hours post-LVAD implantation decreased mean pulmonary artery pressure (mPAP) and increased LVAD flow. However, it did not reduce RVF occurrence, and the most benefit was obtained in patients with higher values of mPAP and low pump flow during weaning from CPB..$^{25,26}$ Sildenafil is a phosphodiesterase-5 (PDE-5) inhibitor commonly used as a selective vasodilator and can lower PVR in persistent pulmonary hypertension post-LVAD implant. ${ }^{27}$

\section{Inotropes}

Milrinone and dobuatmine are the two inotropes approved for use in the United States. Inotropic therapy is used to support an overwhelmed RV during the perioperative period and improve cardiac index and pump flow. Ideally, the patient should be weaned off as soon as the hemodynamics improve. Milrinone is a PDE-5 inhibitor that is both an inotrope and a vasodilator with a longer half-life. Hence, it should be used with caution in patients with renal dysfunction. Dobuatmine is a $\beta-1$ agonist with lesspronounced vasodilatory properties and a very short half-life.

Dopamine and epinephrine are primarily vasopressors with some intrinsic inotropy, and they increase vascular resistance in incremental doses. Their use in the preoperative period has been associated with poor outcomes; thus, vasopressors in general should be avoided in the postoperative period unless a concomitant vasodilatory syndrome exists, thereby mandating its use to maintain a mean blood pressure of $>60 \mathrm{~mm} \mathrm{Hg}$.

\section{Surgical Management}

Intraoperative assessment of RVF and degree of TR is critical in preventing postoperative RV failure. It is common surgical practice to repair moderate-severe TR at the time of CF-LVAD implantation to decrease the chances of a sudden increase in preload. ${ }^{24}$
However, a recent meta-analysis ${ }^{28}$ on concomitant tricuspid valve procedures in patients with severe preoperative TR undergoing LVAD implantation showed worse early postoperative outcomes and no reduction in early death or the need for early right-sided mechanical circulatory support.

In cases where RVF compromises pump flow and a patient does not wean off of cardiopulmonary bypass, a temporary rightheart bypass can be established. This can be accomplished via pulmonary artery-left atrial graft and has been shown to improve RV function. . $^{2,30}$

Intraoperative echocardiography is used to assess biventricular and valvular function, presence of shunts, and detection of cardiac thrombi. Adequate pump function should be confirmed at this time, and if cardiac index is $<2.0$ and central venous pressure is $>$ 20, a temporary RVAD should be considered. ${ }^{12,31}$ Some authors propose that elective BiVAD implantation has better outcomes than unplanned urgent institution of mechanical RV support. ${ }^{12,16,32}$

Finally, pump speed should be set in a range to provide optimal cardiac output, avoiding septal shifts and chamber collapse. This can be achieved intraoperatively, guided by either transesophageal echocardiogram or PAC.

\section{Conclusion}

Right ventricular failure is the most common complication in the immediate postoperative period of LVAD implantation, contributing to significant morbidity. Early recognition and management with aggressive volume removal, inotropic support, and pulmonary vasodilation is essential.

Conflict of Interest Disclosure: The authors have completed and submitted the Methodist DeBakey Cardiovascular Journal Conflict of Interest Statement and none were reported.

Funding/Support: The authors have nothing to disclose.

Keywords: heart failure, heart assist device, right heart dysfunction, cardiac risk factors, outcomes

\section{References}

1. Go AS, Mozaffarian D, Roger VL, et al. Heart disease and stroke statistics--2013 update: a report from the American Heart Association. Circulation. 2013 Jan 1;127(1):e6-e245.

2. Miller LW, Guglin M. Patient selection for ventricular assist devices: a moving target. J Am Coll Cardiol. 2013 Mar 26;61(12):1209-21.

3. Kirklin JK, Naftel DC, Pagani FD, et al. Sixh INTERMACS annual report: a 10,000-patient database. J Heart Lung Transplant. 2014 Jun;33(6):555-64.

4. Kirklin JK, Naftel DC, Stevenson LW, et al. INTERMACS database for durable devices for circulatory support: first annual report. J Heart Lung Transplant. 2008 Oct;27(10):1065-72.

5. Ochiai Y, McCarthy PM, Smedira NG, et al. Predictors of severe right ventricular failure after implantable left ventricular assist device insertion: analysis of 245 patients. Circulation. 2002 Sep 24;106(12 Suppl 1):1198-202.

6. Fukamachi K, McCarthy PM, Smedira NG, Vargo RL, Starling $\mathrm{RC}$, Young JB. Preoperative risk factors for right ventricular failure after implantable left ventricular assist device insertion. Annals Thoracic Surg. 1999 Dec;68(6):2181-4.

7. Wang Y, Simon MA, Bonde P, et al. Decision tree for adjuvant right ventricular support in patients receiving a left ventricular assist device. J Heart Lung Transplant. 2012 Feb;31(2):140-9.

8. Atluri P, Goldstone AB, Fairman AS, et al. Predicting right ventricular failure in the modern, continuous flow left ventricular 
assist device era. Annals Thoracic Surg. 2013 Sep;96(3):857-63; discussion 63-4.

9. Fitzpatrick JR 3rd, Frederick JR, Hsu VM, et al. Risk score derived from pre-operative data analysis predicts the need for biventricular mechanical circulatory support. J Heart Lung Transplant. 2008 Dec;27(12):1286-92.

10. Drakos SG, Janicki L, Horne BD, et al. Risk factors predictive of right ventricular failure after left ventricular assist device implantation. Am J Cardiol. 2010 Apr 1;105(7):1030-5.

11. Matthews JC, Koelling TM, Pagani FD, Aaronson KD. The right ventricular failure risk score a pre-operative tool for assessing the risk of right ventricular failure in left ventricular assist device candidates. J Am Coll Cardiol. 2008 Jun 3;51(22):2163-72.

12. Kormos RL, Teuteberg JJ, Pagani FD, et al. Right ventricular failure in patients with the HeartMate II continuous-flow left ventricular assist device: incidence, risk factors, and effect on outcomes. J Thorac Cardiovasc Surg. 2010 May;139(5):131624.

13. Kukucka M, Stepanenko A, Potapov E, et al. Right-to-left ventricular end-diastolic diameter ratio and prediction of right ventricular failure with continuous-flow left ventricular assist devices. J Heart Lung Transplant. 2011 Jan;30(1):64-9.

14. Topilsky Y, Hasin T, Oh JK, et al. Echocardiographic variables after left ventricular assist device implantation associated with adverse outcome. Circ Cardiovasc Imaging. 2011 Nov;4(6):64861.

15. Ramachandran DP, Luo C, Ma TS, Clark JW. Modeling study of the failing heart and its interaction with an implantable rotary blood pump. Conference proceedings: Annual International Conference of the IEEE Engineering in Medicine and Biology Society. IEEE Engineering in Medicine and Biology Society. Conference 2011;2011: p. 2403-9.

16. Grant AD, Smedira NG, Starling RC, Marwick TH. Independent and incremental role of quantitative right ventricular evaluation for the prediction of right ventricular failure after left ventricular assist device implantation. J Am Coll Cardiol. 2012 Aug 7;60(6):521-8.

17. Hayek S, Sims DB, Markham DW, Butler J, Kalogeropoulos AP. Assessment of right ventricular function in left ventricular assist device candidates. Circ Cardiovasc Imaging. 2014 Mar;7(2):37989.

18. Puwanant S, Hamilton KK, Klodell CT, et al. Tricuspid annular motion as a predictor of severe right ventricular failure after left ventricular assist device implantation. J Heart Lung Transplant. 2008 Oct;27(10):1102-7.

19. Raina A, Seetha Rammohan HR, Gertz ZM, Rame JE, Woo YJ, Kirkpatrick JN. Postoperative right ventricular failure after left ventricular assist device placement is predicted by preoperative echocardiographic structural, hemodynamic, and functional parameters. J Cardiac Failure. 2013 Jan;19(1):16-24.

20. Kato TS, Farr M, Schulze PC, et al. Usefulness of twodimensional echocardiographic parameters of the left side of the heart to predict right ventricular failure after left ventricular assist device implantation. Am J Cardiol. 2012 Jan 15;109(2):246-51.

21. Vivo RP, Cordero-Reyes AM, Qamar U, et al. Increased rightto-left ventricle diameter ratio is a strong predictor of right ventricular failure after left ventricular assist device. J Heart Lung Transplant. 2013 Aug;32(8):792-9.

22. Aissaoui N, Paluszkiewicz L, Martin Gorria G, et al. Assessment of Right Ventricle before the Implantation of Left Ventricular Assist Device by Echocardiographic Parameters (ARVADE). J Heart Lung Transplant. 32(4):S234-S5.

23. Potapov EV, Stepanenko A, Dandel M, et al. Tricuspid incompetence and geometry of the right ventricle as predictors of right ventricular function after implantation of a left ventricular assist device. J Heart Lung Transplant. 2008 Dec;27(12):127581.

24. Slaughter MS, Pagani FD, Rogers JG, et al. Clinical management of continuous-flow left ventricular assist devices in advanced heart failure. J Heart Lung Transplant. 2010 Apr;29(4 Suppl):S1-39.

25. Potapov E, Meyer D, Swaminathan M, et al. Inhaled nitric oxide after left ventricular assist device implantation: a prospective, randomized, double-blind, multicenter, placebo-controlled trial. J Heart Lung Transplant. 2011 Aug;30(8):870-8.

26. Kukucka M, Potapov E, Stepanenko A, et al. Acute impact of left ventricular unloading by left ventricular assist device on the right ventricle geometry and function: effect of nitric oxide inhalation. J Thoracic Cardiovasc Surg. 2011 Apr;141(4):1009-14.

27. Tedford RJ, Hemnes AR, Russell SD, et al. PDE5A inhibitor treatment of persistent pulmonary hypertension after mechanical circulatory support. Circ Heart Fail. 2008 Nov; 1(4):213-9.

28. Robertson JO, Grau-Sepulveda MV, Okada S, et al. Concomitant tricuspid valve surgery during implantation of continuous-flow left ventricular assist devices: a Society of Thoracic Surgeons database analysis. J Heart Lung Transplant. 2014 Jun;33(6):60917.

29. Spillner J, Amerini A, Hatam N, et al. Pulmono-atrial shunt and lung assist to treat right ventricular failure. Frontiers in Bioscience. 2011 Jan; 16:2342-51.

30. Argiriou M, Mikroulis D, Sakellaridis T, Didilis V, Papalois A, Bougioukas $\mathrm{G}$. Acute pressure overload of the right ventricle. Comparison of two models of right-left shunt. Pulmonary artery to left atrium and right atrium to left atrium: experimental study. J Cardiothorac Surg. 2011 Oct 19;6:143.

31. Patlolla B, Beygui R, Haddad F. Right-ventricular failure following left ventricle assist device implantation. Curr Opin Cardiol. 2013 Mar;28(2):223-33.

32. Fitzpatrick JR 3rd, Frederick JR, Hiesinger W, et al. Early planned institution of biventricular mechanical circulatory support results in improved outcomes compared with delayed conversion of a left ventricular assist device to a biventricular assist device. J Thorac Cardiovasc Surg. 2009 Apr;137(4):971-7. 